M. Priya* and R. Kalpana

\title{
Log Posterior Approach in Learning Rules Generated using N-Gram based Edit distance for Keyword Search
}

DOI 10.1515/jisys-2016-0067

Received May 16, 2016; previously published online April 13, 2017.

\begin{abstract}
Challenging searching mechanisms are required to cater to the needs of search engine users in probing the voluminous web database. Searching the query matching keyword based on a probabilistic approach is attractive in most of the application areas, viz. spell checking and data cleaning, because it allows approximate search. A probabilistic approach with maximum likelihood estimation is used to handle real-world problems; however, it suffers from overfitting data. In this paper, a rule-based approach is presented for keyword searching. The process consists of two phases called the rule generation phase and the learning phase. The rule generation phase uses a new technique called N-Gram based Edit distance (NGE) to generate the rule dictionary. The Turing machine model is implemented to describe the rule generation using the NGE technique. In the learning phase, a log model with maximum-a-posterior estimation is used to select the best rule. When evaluated in real time, our system produces the best result in terms of efficiency and accuracy.
\end{abstract}

Keywords: Probabilistic search, turing machine, edit distance, n-gram, rule generation, MAP.

\section{Introduction}

Search engines play a very important role in query processing and data extraction from the web. The given query/keyword can be effectively processed by the engines, and the documents that are similar to the original given query will be retrieved from the database to the user. The engine applies similarity functions that are used to find the similarity between two parameters or between two strings, including the given query and the document, which is the web database. Most of the search engines use edit distance-based similarity functions $[4,5]$, and $93 \%$ of the errors are corrected [16]. Increasing the web access causes the search engines to retrieve the documents from the heterogeneous environment [3]. For example, to answer the query that contains book information, the search engines have to retrieve the documents from different domains such as a publication, book stall, author, etc. The search engines need to improve the efficiency of document retrieval, compared with the homogeneous environment. Moreover, they have to consider the interrelationship between words to produce accurate results.

Similarity measurement plays an important role in spell checking $[6,17]$. The spell-checking process consists of detection of error, candidate generation mechanism, and the selection of the context-based candidate for correction. The error model is introduced to handle the error detection and candidate generation mechanisms. This is helpful for finding the list of most likely error words for the given word. The language model or source model is established to select the best candidate for correction. It helps to list the most likely corrected word for the given word.

The first stochastic model for string edit distance with a learning algorithm is described by Ristad and Yianilos [9]. It provides an efficient algorithm to automatically learn the edit distance cost of similar strings.

*Corresponding author: M. Priya, Bharathiyar College of Engineering and Technology, Department of Computer Science and Engineering, Karaikal, Puduchery, India, e-mail: shyamnithy@gmail.com

R. Kalpana: Pondicherry Engineering College, Department of Computer Science and Engineering, Pillaichavady, Puducherry, India 
A generalized framework called expectation and maximization is used to maximize the probability of the training data and optimize the parameters according to the model. The expectation step is used to accumulate the expectations of edit operations applied to generate the string pairs, and the maximization step is used to maximize the parameter values.

This paper introduces a new rule-based model for finding the similarity of the query keyword with the databases. The model receives the training set and applies the n-gram-based edit distance (NGE) mechanism to find the similarity and generate the transformation rules. The importance of each rule is calculated approximately by applying the conditional probability function. Maximum-a-posterior (MAP) estimation is used to estimate the probability and to maximize it. The paper is organized as follows: Section 2 discusses the related work. Section 3 defines a new model called log posterior model and describes NGE techniques using the Turing machine. Section 4 presents an experimental evaluation against a real-time system. The final section concludes.

\section{Related Work}

Arasu et al. introduced a token-based approach [1] for matching the strings from a homogeneous domain. The strings can be matched based on identity and alternative relationship. Rule selection is the main constraint in this model. A domain-independent approach [21] is introduced to learn the similar objects from a heterogeneous resource. The active supervised learning technique is used to learn the objects. However, it suffers from the problem of user intervention. A lexicon-based unsupervised learning algorithm [10] is designed to match the two strings. In this model, the training pair is derived after the match is found out. This mechanism is similar to the supervised learning algorithm [9], except that the training pairs are given directly.

The approaches used to learn the strings in the probabilistic model are generative and discriminative. A language model based on a generative approach [16] is introduced to reduce the error rate in spelling correction. In this model, the Levenshtein-based edit distance technique is used to generate the rules. The first stochastic learning model for string edit distance is introduced by Ristad and Yianilos [19]. It is based on two edit distances called Viterbi distance and stochastic distance for finding the similarity of string pairs. The conditional probability and joint probability functions are used to calculate the edit cost. The Levenshtein edit distance model $[5,10]$ is also used to find the edit distance sequences. The rules are classified based on forward and backward classification algorithm. A discriminative-based approach introduced a log linear model [23] and logistic regression model [20] to learn the strings. Rules can be generated either based on the weighted edit distance technique [2, 19, 21] or n-gram-based technique [4, 13]. In most of the cases, the inductive principle is used to learn the parameters of an edit distance. In case of the probabilistic approach [12], maximization of the likelihood principle is used to learn the edit parameters. However, this technique suffers from the problem of overfitting the data. Bellet et al. [5,6] proposed a new method for the learning edit similarity function based on the loss minimization method.

Boyer et al. [7] and McCallum et al. [14] introduced a finite state transducer to learn the edit distance [3, 8, 11] based on a probabilistic framework. The probabilistic work model can also be applied for record linkage. It requires multiple passes over the database to review the data and to improve the accuracy of the transformation. Most of the techniques are domain specific, which consists of a general comprehensive set of transformations. However, these techniques are not suitable for combining the record linkage from heterogeneous resources.

Initially, a predefined set of transformation rules obtained from online resources is used. Later, a concept of generating the rules automatically is used; however, the scope of this method is limited to a homogeneous domain. Moreover, there is an issue of generating rules for the string pairs containing more than one word. Generally, string pairs can be categorized into words and the transformation rule is created for each word based on the syntactic structure of the string. The transformation rules are learned [18, 20, 22, 24, 25] to generate top-k transformation rules.

In existing methods, the log linear-based probabilistic approach [15] is the best method to learn the model, as it considers the description of the independent events and it is the most suitable model for handling high 
variance. The edit distance-based log linear model with maximum likelihood estimation (MLE) [23] is presented to learn rules. It has the following drawbacks: (i) MLE suffers from overfitting data, underfitting data, and bias/high variance; (ii) the maximum number of rule generations is limited; and (iii) edit distance-based rule generation is limited by the threshold. In this paper, an n-gram-based log linear model with MAP estimation is proposed to generate and learn the rules in order to produce better accuracy and efficiency.

\section{Log Posterior Model}

The techniques to generate the transformation rules for the misspelled string pair can be categorized into three ways: edit distance, substitution, and n-gram-based method. In the edit distance method, given the string pair $\left(s_{i}, t_{i}\right)$, where $s_{i}$ is the source string that can be transformed to the correct string $t_{i}$ by applying different edit operations, namely insertion, deletion, etc., and the different edit sequence between the given pair is produced by the alignment technique. Sequence alignment, global alignment, and local alignment are the different types of alignment algorithms available to produce better edit alignment. After producing the better alignment, match the pair of $s_{i}$ and $t_{i}$, and then produce the rules if there is a mismatch or gap.

For example, the given pair is ("basicly", "basically"). The alignment between two strings are as shown in Figure 1.

In the substitution-based rule generation method, the common prefix and common suffix between the given pair are found out and removed. The string portion that is uncommon is extracted, and then the substitution rules are employed. For the given pair ("basicly", "basically"), the common prefix (cp) is "basic" and the common suffix (cs) is "ly". The given pair is expressed as cp Ø cs and cp al cs. The uncommon pair $\emptyset$, al is extracted and the rule is generated. The edit-based method does not limit the number of rules generated. The substitution method works well if there is a common prefix and suffix; otherwise, it will simulate the edit distance model. Moreover, the substitution-based mechanism does not specify any standard mechanisms to eliminate the common prefix and suffix.

The $\mathrm{n}$-gram model is the language model that assigns the probability to each sequence. The $\mathrm{n}$-gram has the size of one, called unigram, and size two, called bigram, etc. The bigram model produces better efficiency when compared with trigram. The new framework has the combination of the n-gram model and the edit operations to produce the character-level transformation rules. For word level, an abbreviation dictionary or relationship among the words is considered. The framework consists of two phases, namely

1. Rule generation phase;

2. Learning phase.

\subsection{Rule Generation Phase}

The transformation technique, such as character-level transformation and word-level transformation, is used to transform a string to another string. From the training data, rules are generated by applying the NGE technique, as shown in Figure 2.

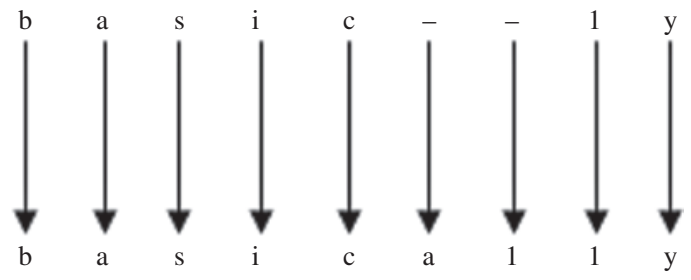

Figure 1: Edit-Based Sequence Alignment. 


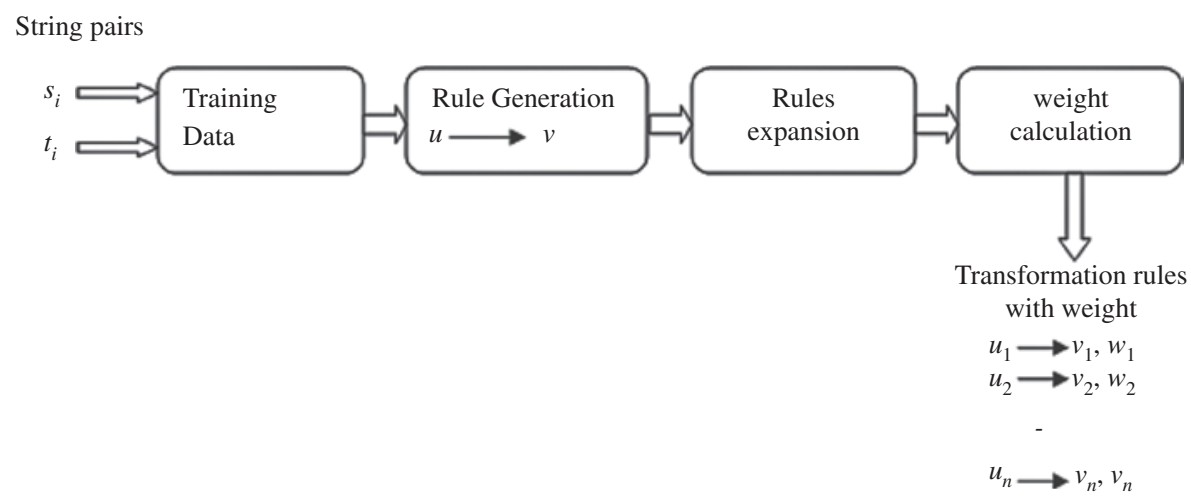

Figure 2: Rule Generation Phase.

The generated rules are in the form of $u \rightarrow v$, and are expanded by adding their contexts. The number of characters added to the contexts is limited. If the transformation rules for a string pair cannot be generated by the n-gram model, then the relationship between the string pairs can be found by using an abbreviation dictionary or by using the mining rules. For each rule, the weight $\left(w_{i}\right)$ is calculated based on conditional probability distribution.

The rule generation algorithm for the given training pair is described in Figure 3. The algorithm will perform word-level transformation if the given pair belongs to the abbreviation dictionary; otherwise, the associative rules apply if there is a relationship between two words. If the given pair is a single misspelled word, then character-level transformation using the NGE algorithm, shown in Figure 4, is applied.

The transformation rules are generated for the given pair. There is a possibility of having multiple rules, and the number of rules generated with context is not restricted.

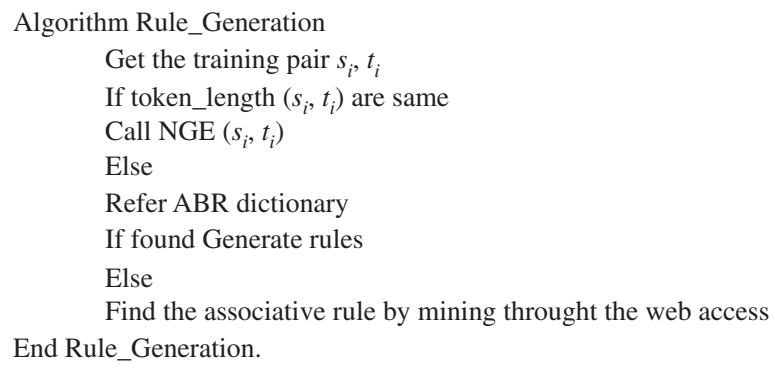

Figure 3: Rule Generation Algorithm.

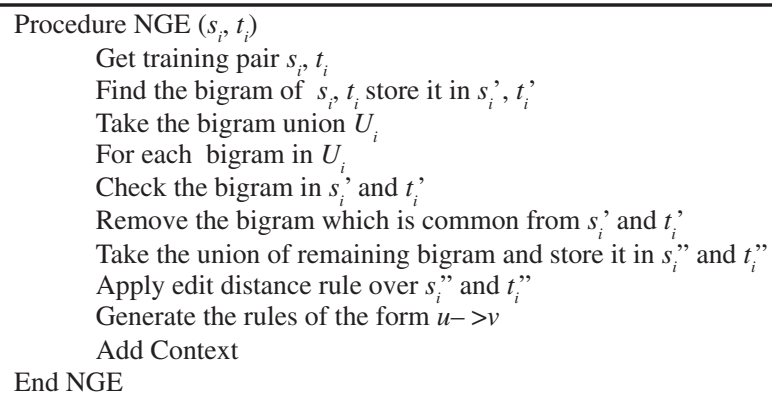

Figure 4: NGE Algorithm. 


\section{(a) Implementation of NGE using Turing machine}

The Turing machine with multiple tapes is utilized to implement the NGE algorithm. The machine has the following components: a finite control, three tapes, and the corresponding tape heads. Each tape consists of two tracks for storing bigrams. The bigrams of given training pairs $s_{i}$ and $t_{i}$ are stored in tape 1 and tape 2 , and the bigram union of the pair $s_{i}$ and $t_{i}$ is stored in tape 3 . The machine for the given pair ("basicly", "basically") is shown in Figure 5.

The bigram in tape 3 is compared with tape 2 and tape 1 . If the bigram does not exist in any one of the tapes, then move the corresponding tape head to the right end, append the bigram, and then return the tape head to its original position. Move all the tape head to the one cell right. Repeat this process until tape head 3 reaches the bigram with \# symbol. Generate the rules by taking the content of tape head 1 as left side and tape head 2 as right side, then move the tape head one cell right. The common characters in the left and right sides are removed to generate the rules with its context. Rules generated for the given pair are as follows:

$$
c l \rightarrow c a, \emptyset \rightarrow a l, \emptyset \rightarrow l l .
$$

\section{(b) Weight calculation}

The rule generation phase will generate multiple rules for transforming each input string to output string. The occurrence of the $u$ for the given $v$ is calculated by conditional probability, as given in Eq. (1):

$$
P(u \mid v)=\frac{P(u) P(v \mid u)}{P(v)} .
$$

If $u$ and $v$ contains more than one character, then the weight is calculated by using joint probability, as given in Eq. (2):

$$
P\left(u=x_{1} x_{2}, \ldots, x_{n}\right)=P\left(x_{1} \mid x_{2} \ldots x_{n}\right) P\left(x_{2} \mid x_{3} \ldots x_{n}\right) \ldots P\left(x_{n}\right) .
$$

At the end of the rule generation phase, the transformation rules with weights are generated in the form given in Eq. (3):

$$
u_{i} \rightarrow v_{i}, w_{i}
$$

\subsection{Learning Phase}

It is impossible to predict the best rules to transform the strings. In order to select the best rules, a learning model is required. In our method, a log linear model with MAP estimation is proposed to learn the model. In the learning phase, the rules are learned and classified by using the binary rule classification algorithm. The learning phase is shown in Figure 6.

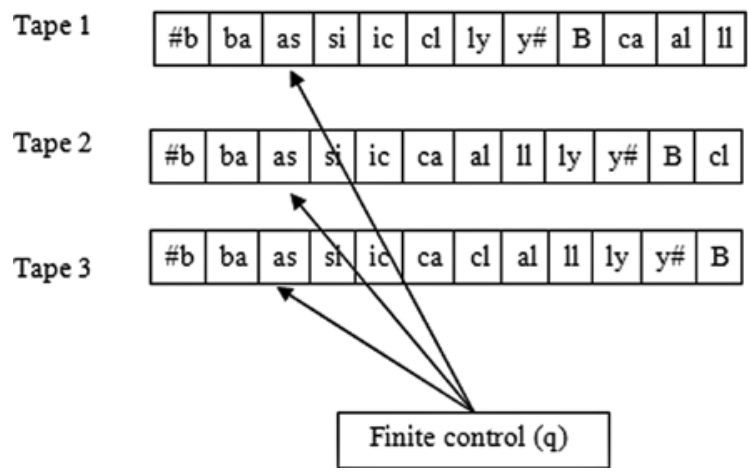

Figure 5: Implementation of NGE using Turing Machine. 


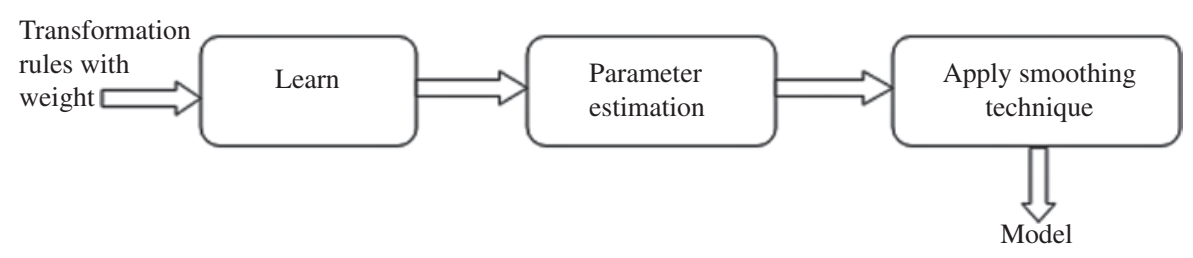

Figure 6: Learning Phase.

Let $T_{r}\left(s_{i}, t_{i}\right)$ be the transformation rules created for the string pair $\left(s_{i}, t_{i}\right)$. The mapping between the input string, output string, and the transformation rules are expressed with conditional probability. The linear model defines the probability for the $T_{r}\left(s_{i}, t_{i}\right)$, and $t_{i}$ for the given $s_{i}$ is given in Eq. (4):

$$
P\left(t_{i}, T_{r}\left(s_{i}, t_{i}\right) \mid s_{i}\right)=\frac{\operatorname{Exp}\left(\sum_{i=1}^{k} w_{i}\right)}{z\left(s_{i}\right)},
$$

where $K$ is the number of elements in weight vector. The value of this probability will always remain positive. The normalizing constant is calculated as per Eq. (5):

$$
z\left(s_{i}\right)=\sum_{t_{i}^{\prime}} \operatorname{Exp}\left(\sum_{i=1}^{k} w_{i}\right) .
$$

The $\log$ function $L(\omega)$ is defined in Eq. (6), and it is maximized by applying MAP estimation and it is denoted by $\omega_{\mathrm{MAP}}^{*}$, as shown in Eq. (7):

$$
\begin{gathered}
P\left(t_{i} \mid s_{i}\right)=\sum_{T_{r}\left(s_{i}, t_{i}\right)} P\left(t_{i}, T_{r}\left(s_{i}, t_{i}\right) \mid s_{i}\right) \\
L(\omega)=\sum_{k} \log P\left(t_{i} \mid s_{i}\right), \\
\omega_{\text {MAP }}^{*}=\operatorname{argmax}\left(P\left(t_{i} \mid s_{i}\right)\right) \\
=\operatorname{argmax} \sum \log P\left(s_{i}\right)+\sum_{j} \log \left(P\left(t_{i} \mid s_{i}\right)\right),
\end{gathered}
$$

where $\log P\left(s_{i}\right)$ denotes prior probability. The parameters can be optimized using the limited BFGS (BroydenFletcher-Goldfarb-Shanno algorithm) smoothing technique algorithm.

\section{Experimental Evaluation}

An Intel Pentium processor with frequency of $2.10 \mathrm{GHz}$ is used to conduct the experiment. A synthetic data set with 100 misspelled words is created to test the experiment. The words are collected from Peter Norvig Dictionary, which consists of words from Project Gutenberg, Wiktionary, and British National Corpus. The efficiency and accuracy of the system is evaluated. The performance of the NGE method is compared with edit distance and the substitution method.

The chart given in Figure 7 represents the comparison of running time achieved in various methods, namely edit distance, substitution, and NGE. The $\mathrm{x}$-axis represents word length, and the y-axis represents running time in milliseconds.

The results show that the NGE method gives a reduced running time compared to that of the edit distance method and substitution method. The reduced running time is due to the reduced word length where the rule 


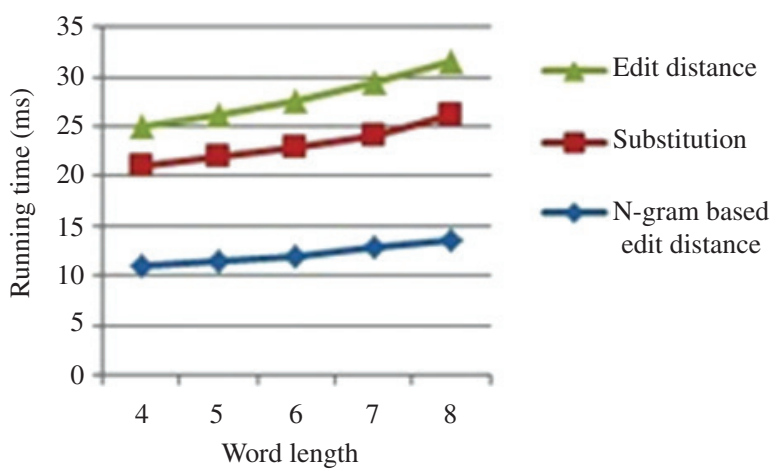

Figure 7: Comparison of the Running Time in the Various Methods.

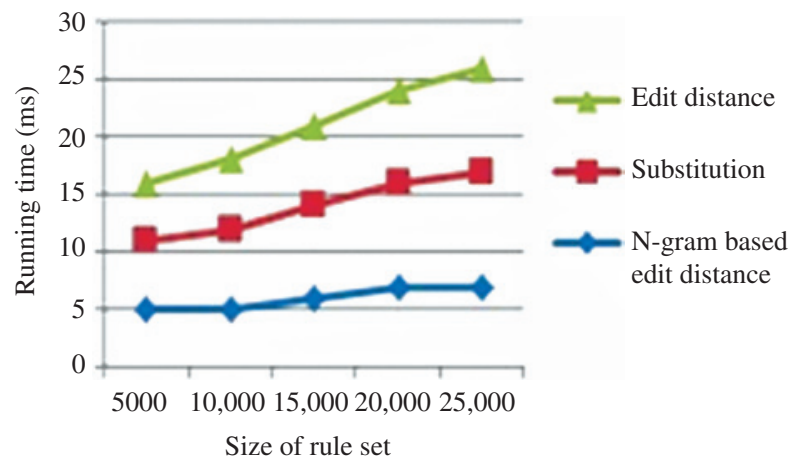

Figure 8: Effects of Size of Rule Set in Various Methods.

generation algorithm was applied. The NGE removes the common bigrams, so that the length of the word is reduced.

The chart given in Figure 8 represents the effects of the size of rule set in various methods. The $x$-axis represents the size of rule set, and the y-axis represents the running time in milliseconds.

The reason for the reduced running time in the NGE method compared to that of other methods is that the running time depends on the number of matches found in the rule set. If the number of matches for the keyword increases, then the running time also increases. The NGE method limits the number of rules generated, so that the number of matches is also restricted.

The running time against the number of rules applicable for the word is represented in the chart given in Figure 9. The x-axis represents the maximum number of applicable rules, and the y-axis represents the running time in milliseconds.

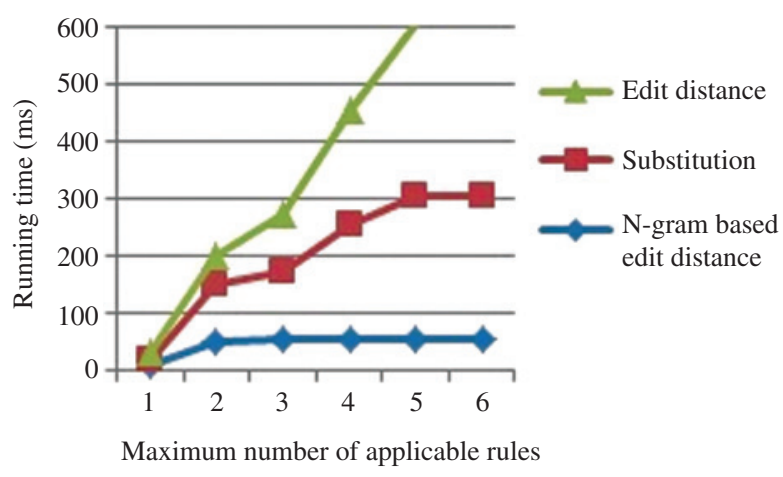

Figure 9: Effect of Number of Rules in Various Methods. 


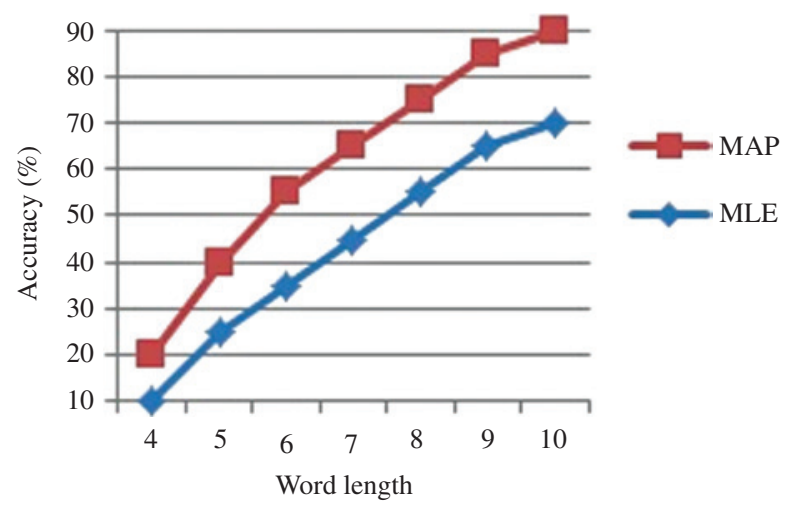

Figure 10: Accuracy between MLE and MAP.

If the number of rules applied decreases, then the running time also decreases. The NGE method generates a smaller amount of rules with context for each training set, so that this method requires only less number of rules to be applied for correction compared to that of other methods. It causes reduced running time.

The accuracy of this method depends on two factors: (i) associative rules for word and (ii) probability value assigned for each rule. It is determined by the best rule selected for the transformation. The MAP estimation is used to estimate the probability associated with each rule. The MAP method works better and provides the best accurate result than MLE, as shown in Figure 10.

Often, MLE overfits the data and does not generalize well. However, MAP generalizes well by allowing the prior distribution. Each rule in the system is associated with the MAP estimated probability value. The search engines can select the appropriate rule to correct the given mistaken keyword and also can improve the search space.

\section{Conclusion}

In this paper, two improvements over the existing system is discussed. For the rule generation phase, the existing edit distance is replaced with a new technique, NGE, which produces better performance when the word length and the number of errors are greater. The rule for candidate correction is selected based on the probabilistic value associated with each rule. The MAP estimation model is used, which overcomes the overfitting problem. This model can be applied in databases, search engines, etc., for the keyword search process, and it produces better efficiency and accuracy.

\section{Bibliography}

[1] A. Arasu, S. Chaudhuri and R. Kaushik, Learning string transformations from examples, in: Proc. VLDB Endow., vol. 2, pp. 511-525, August 2009. Available at: https://www.researchgate.net/publication/220538475.

[2] L. R. Bahl and R. Jelinek, Decoding for channel for insertion, deletion, substitution application to speech character recognition, IEEE Trans. Inform. Theory (IT 21) 4 (1975), 404-411.

[3] M. F. Balcan and A. Blum, On a theory of learning with similarity functions, in: Proceedings of the 23rd International Conference on Machine Learning, Pittsburgh, PA, 2006.

[4] A. Behm, S. Ji, C. Li and J. Lu, Space-constrained gram-based indexing for efficient approximate string search, in: Proceedings of the 2009 IEEE International Conference on Data Engineering, ser. ICDE '09, pp. 604-615, IEEE Computer Society, Washington, DC, USA, 2009.

[5] A. Bellet, A. Habrard and M. Sebban, An experimental study on learning with good edit similarity functions, in: International Conference on Tools with Artificial Intelligence United States (ICTAI), pp. 181-188, 2011. 
[6] A. Bellet, A. Habrard and M. Sebban, Good edit similarity learning by loss minimization, Mach. Learn. J. 89 (2012), 5-35.

[7] L. Boyer, A. Habrard, F. Muhlenbach and M. Sebban, Learning string edit similarities using constrained finite state machines, in: Conférence Francophone surl' Apprentissage Automatique, pp. 37-52, Porquerolles, France, 2008.

[8] E. Brill, Transformation-based error-driven learning and natural language processing: a case study in part-of-speech tagging, Comput. Linguist. 21 (1995), 543-565.

[9] E. Brill and R. C. Moore, An improved error model for noisy channel spelling correction, in: Proceedings of the 38th Annual Meeting on Association for Computational Linguistics, ser. ACL '00, pp. 286-293, Association for Computational Linguistics, Morristown, NJ, USA, 2000.

[10] A. W. Hauser and K. U. Schulz, Unsupervised learning of edit distance weights for retrieving historical spelling variations, in: Proceedings of the First Workshop on Finite-State Techniques and Approximate Search, pp. 1-6, Borovets, Bulgaria, 2007.

[11] A. B. Hillel and D. Weinshall, Learning distance function by coding similarity, in: Proceedings of the 24th International Conference on Machine Learning, Corvallis, OR, 2007.

[12] A. Islam and D. Inkpen, Real-word spelling correction using Google Web IT 3-grams, in: Proceedings of the 2009 Conference on Empirical Methods in Natural Language Processing, ser. EMNLP '09, pp. 1241-1249, Association for Computational Linguistics, Morristown, NJ, USA, 2009.

[13] M. Li, M. Zhu, Y. Zhang and M. Zhou, Exploring distributional similarity based models for query spelling correction, in: $A C L$, pp. 1025-1032, Sydney, July 2006.

[14] A. McCallum, K. Bellare and F. Pereira, A conditional random field for discriminatively-trained finite-state string edit distance, in: Proceedings of the 21st Conference on Uncertainty in Artificial Intelligence, ser. UAl '05, pp. 388-395, 2005.

[15] M. Nauhaus and H. Bunke, A probabilistic approach to learning costs for graph edit distance, in: Proceeding of 17th International Conference on Pattern Recognition, 2004.

[16] N. Okazaki, Y. Tsuruoka, S. Ananiadou and J. Tsujii, A discriminative candidate generator for string transformations, in: Proceedings of the Conference on Empirical Methods in Natural Language Processing, ser. EMNLP'08, pp. 447-456, Association for Computational Linguistics, Morristown, NJ, USA, 2008.

[17] J. Oncina and M. Sebban, Learning unbiased stochastic edit distance in the form of a memory less finite-state transducer, in: Workshop on Grammatical Inference Applications: Successes and Future Challenges, Pattern Recognition and Artificial Intelligence Group, Alicante, Spain, 2005.

[18] S. Patro and W. Wang, Learning top-k transformation rules, in: Proceedings of the 22nd International Conference on Database and Expert Systems Applications, DEXA 2011, Toulouse, France, pp. 172-186, 2011.

[19] E. S. Ristad and P. N. Yianilos, Learning string-edit distance, IEEE Trans. Pattern Anal. Mach. Intell. 20 (1008), 522-532.

[20] R. Singh and S. Gulwani, Learning semantic string transformations from examples, in: Technical Report MSR-TR-2012-5, Microsoft Research, 2012.

[21] S. Tejada, C. A. Knoblock and S. Minton, Learning domain independent string transformation weights for high accuracy object identification, in: Proceedings of the Eighth ACM SIGKDD International Conference on Knowledge Discovery and Data Mining, pp. 350-359, ACM, New York, USA, 2002.

[22] R. Vernica and C. Li, Efficient top-k algorithms for fuzzy searching string collections, in: Proceedings of the First International Workshop on Keyword Search on Structured Data, ser. KEYS '09, pp. 9-14, ACM, New York, NY, USA, 2009.

[23] Z. Wang, G. Xu, H. Li and M. Zhang, A probabilistic approach to string transformation, IEEE Trans. Knowl. Data Eng. 26 (2013) 1063-1075.

[24] B. Wu, P. Szekely and C. A. Knoblock, Learning data transformation rules through examples: preliminary results, in: IIWEB '12, Scottsdale, AZ, USA, 2012.

[25] B. Wu, P. Szekely and C. A. Knoblock, Learning transformation rules by examples, in: Proceedings of the Twenty-Sixth AAAI Conference on Artificial Intelligence, Association for the Advancement of Artificial Intelligence, IIWEB ' 12 Scottsdale, AZ, USA, 2012. 